\title{
Comparison of samples with flat bottom holes and with hidden occlusions using flash thermography
}

\author{
by B. Oswald-Tranta, R.Schmidt, T.Grandl
}

Chair of Automation, University of Leoben, Austria, beate.oswald@unileoben.ac.at

\begin{abstract}
FEM simulation models have been setup to compare temperature distribution over flat bottom hole defects and occlusions filled with air or with other materials, as e.g. with aluminum. The temperature distribution is evaluated with PPT and TSR techniques. It is investigated, whether it is possible to distinguish a flat bottom hole defect and an air gap inside a material, and what is the difference in the phase and second derivative images depending on the type of the enclosed material. Plastic samples with air and with metal inclusions were created with 3D printing, tested with flash thermography and the results compared with the simulated ones.
\end{abstract}

Keywords: thermography, flash heating, defect detection, PPT, TSR

\section{Introduction}

To demonstrate the capabilities and to investigate the limits of thermographic testing often samples with artificial defects are used. For subsurface defects usually samples with flat bottom holes (FBH) are created, as they are simple to manufacture. Even if real defects in most of the cases are enclosed occlusions, it is difficult to produce such kind of artificial defects with a given geometry. Therefore the main goal of these current investigations is, whether thermographic results obtained for $\mathrm{FBH}$ samples give a reliable information also about the detectability of hidden occlusions, and whether there are significant or any differences between these two kind of defects.

In the first part of the paper the difference between flash thermography results for samples with flat bottom holes and with occlusions containing air are investigated. In further step also the influence of the defect height and its lateral size are taken into consideration. In a further step aluminium is placed inside the occlusion instead of air, and its influence on the thermographic results is tested. FEM simulation models have been setup to calculate the temperature distribution after a short pulse heating. The calculated temperature is evaluated with PPT and TSR techniques.

3D printing makes possible to create samples with $\mathrm{FBH}$ and also with occlusions, where inside the hole air or other materials can be enclosed. Different plastic samples have been printed in this way, and the measurement results are compared with the simulated ones.

\section{Temperature above a subsurface defect}

For testing a sample with flash thermography in reflection mode, first the surface is heated with a short flash pulse, which is absorbed and dissipated into heat. The temperature of the cooling surface is recorded with an infrared camera and the temperature versus time function is usually plotted in a double logarithmical scale. The cool down phase for a sample with a subsurface defect can be divided into the following ranges, see also in Fig.1:

I: Under ideal conditions (Dirac delta heating and adiabatic conditions) the temperature decreases at the sound surface as well as above the defect with $1 / \sqrt{t}$ [1], which means a straight line with a slope of $-1 / 2$ in a double logarithmical scale. This is the typical behaviour of a semi-infinite body, and the sound surface and also the part above a subsurface defect behaves as a semi-infinite body shortly after the flash heating. As the heat reaches the defect depth, it starts to accumulate above the failure. This time is marked with $t_{w}$, where

$$
t_{w}=\frac{d^{2}}{\pi \kappa}
$$

and $d$ is the defect depth and $\kappa$ is the thermal diffusivity of the material.

II: Hence the heat is trapped between the defect and the surface of the sample, the temperature remains mainly constant with the value of 


$$
T_{d}=\frac{Q \kappa}{\lambda d}
$$

where $Q$ is the heat amount dissipated by the sample and $\lambda$ is the thermal conductivity of the material.

III: Due to the lateral extension of the defect, the trapped heat flows laterally away and the temperature above the defect decreases again. This time is denoted as $t_{c}$, and the temperature contrast between the defect and the sound surface is here the largest. This time depends mainly on the size of the defect.

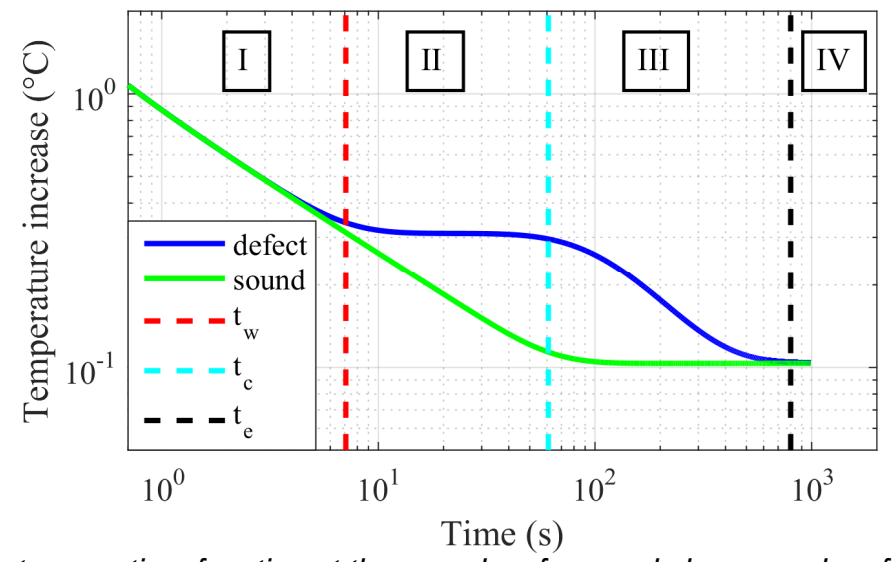

Figure 1: Temperature vs. time function at the sound surface and above a subsurface defect, divided into four regions.

IV: The temperature over the whole sample is homogeneous: due to the lateral heat flow the trapped heat above the defect is decayed and a homogeneous temperature at the whole front surface is achieved. The start of this region is denoted with $t_{e}$ in Fig.1. How $t_{c}$ and $t_{e}$ can be estimated from the material parameters and from the geometry of the defect, will be investigated in a separate publication.

\section{Simulation models for subsurface defects}

In order to investigate the difference between a FBH defect and a fully included subsurface defect, finite element simulation models have been setup. For the calculations the multiphysics simulator ANSYS [2] has been used. Fig. $2 \mathrm{a}$ and $2 \mathrm{~b}$ show both models, where the remaining wall thickness above the defects in both cases is $d=2 \mathrm{~mm}$ and the defect diameter is $D=20 \mathrm{~mm}$. For the simulations typical plastic material parameters have been used, summarized in Table 1. The occlusion in Fig.2b has a height of $d_{g}=2 \mathrm{~mm}$ and it contains air.
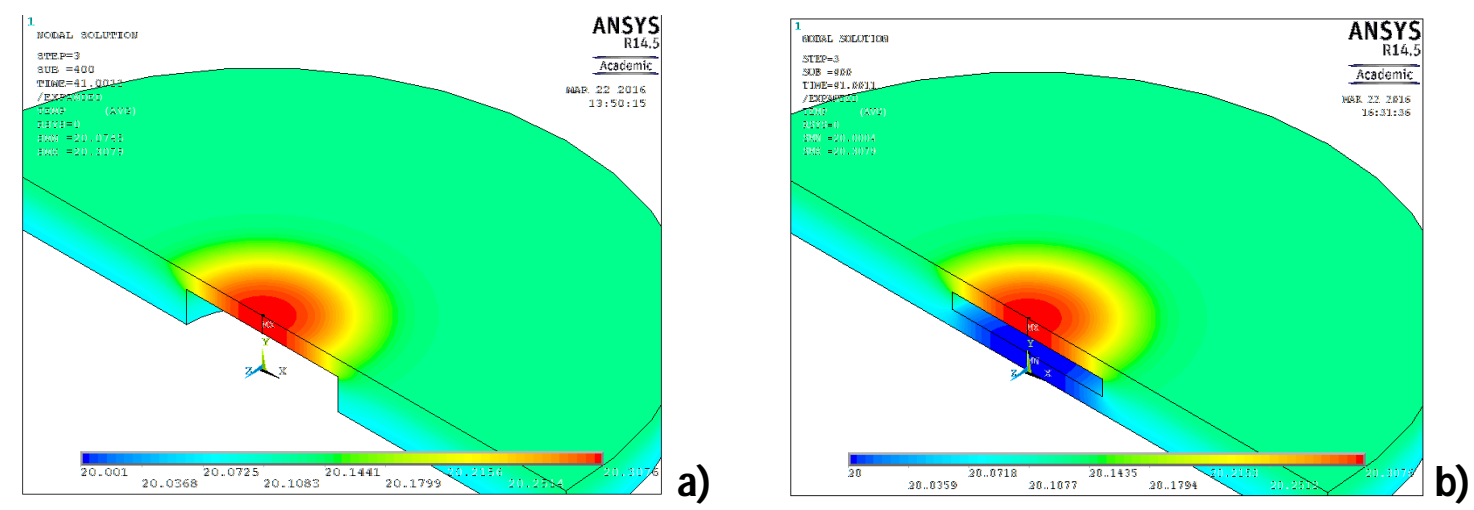

Figure 2: Finite element simulation models $\left(d=2 \mathrm{~mm}, d_{\text {sound }}=6 \mathrm{~mm}, D=20 \mathrm{~mm}\right)$; $a$ : flat bottom hole defect; $b$ : inclusion containing air with a height $d_{g}=2 \mathrm{~mm}$

Since a flash thermography measurement is carried out in reflection mode, only the temperature at the front surface is measured. As in Fig.2 can be observed, these temperature distributions are in both cases mainly identical. The difference occurs only at the back side of the sample, which is not observed in reflection mode: in the case of the occlusion (Fig.2b) the heat flows only slowly into the region below the defect, and there is the temperature lower than at the sound part of the back surface.

Several simulation models have been used varying the height of the occlusion. Fig. 3 compares the temperature distribution in cross section for a model of flat bottom hole defect (Fig.3a) and an occlusion with a height $d_{g}=0.2 \mathrm{~mm}$. It 
can be well observed how the heat flows into the region below the occlusion, but this has hardly any effect on the surface temperature.

Table 1: Parameters used in the simulations

\begin{tabular}{|l|c|c|c|c|c|}
\hline Material & $\begin{array}{c}\text { Thermal } \\
\text { conductivity } \lambda \\
{\left[\mathrm{W} \mathrm{m}^{-1} \mathrm{~K}^{-1}\right]}\end{array}$ & $\begin{array}{c}\text { Thermal } \\
\text { diffusivity } \kappa \\
{\left[\mathrm{m}^{2} \mathrm{~s}^{-1}\right]}\end{array}$ & $\begin{array}{c}\text { Time duration } \\
t_{\text {end }} \\
{[\mathrm{s}]}\end{array}$ & $\begin{array}{c}\text { Sampling } \\
\text { frequency } f_{s} \\
{[\mathrm{~Hz}]}\end{array}$ & $\begin{array}{c}t_{w} \text {, if } d=2 \mathrm{~mm} \\
{[\mathrm{~s}]}\end{array}$ \\
\hline plastic & 0.29 & $1.8 \mathrm{e}-7$ & 990 & 10 & 7 \\
\hline steel & 40 & $1.16 \mathrm{e}-5$ & 15 & 660 & 0.11 \\
\hline aluminum & 218 & $1.2 \mathrm{e}-4$ & & & \\
\hline
\end{tabular}
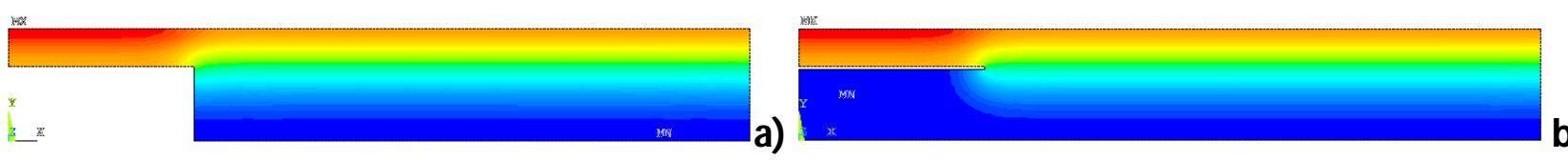

Figure 3: Cross-sections of the half FEM models $\left(d=2 \mathrm{~mm}, d_{\text {sound }}=6 \mathrm{~mm}, D=20 \mathrm{~mm}\right)$; : flat bottom hole defect; $b$ : inclusion containing air with a height $d_{g}=0.2 \mathrm{~mm}$

Fig.4 compares the temperature versus time functions for the midpoint above the defect and for the sound surface for these three models:

- $\quad$ flat bottom hole model (the same model as in Fig.2a and Fig.3a);

- $\quad$ model with an air inclusion with a height $2 \mathrm{~mm}$ (also shown in Fig.2b);

- $\quad$ and with $0.2 \mathrm{~mm}$ air occlusion (cross-section of this model is shown in Fig.3b).

In regions I and II the temperature functions of all the three models are identical, as here the heat flow just reaches the top of the defect and it accumulates above it, without any influence what is below the top of the defect. A slight difference occurs in region III. As the trapped heat above the defect flows laterally away, the heat flows also around the occlusion into the region below it. The temperature decreases slightly quicker in the case of occlusions than in the case of bottom hole.
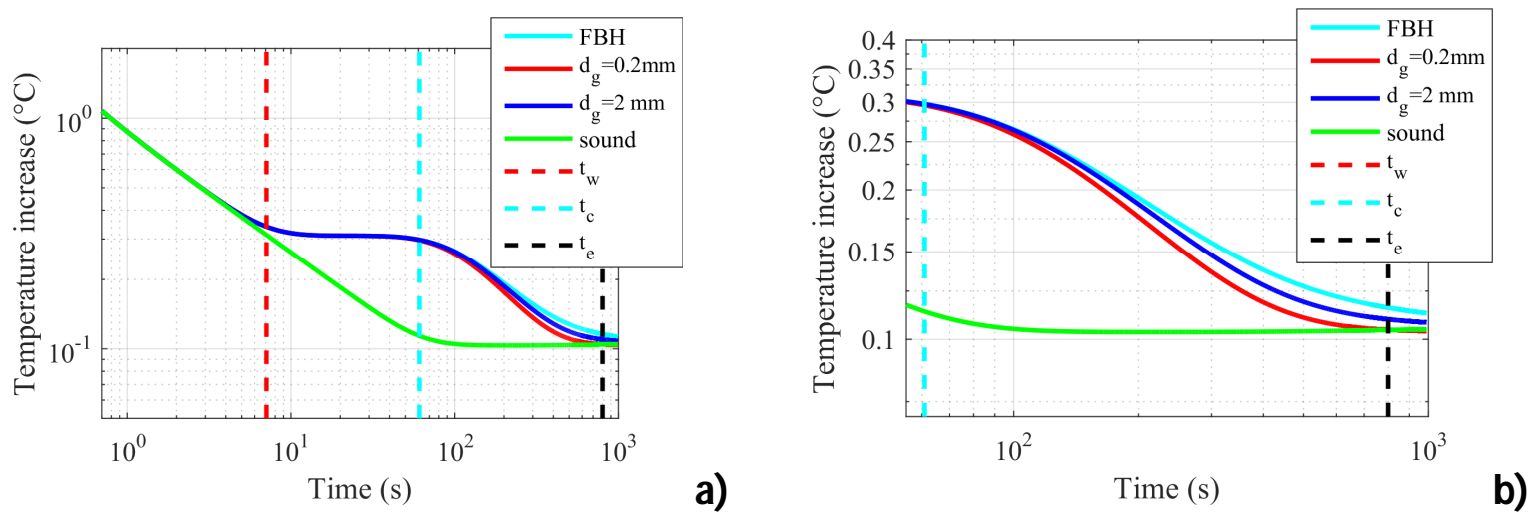

Figure 4: a: Temperature versus time plot in double logarithmical scale, comparing the temperature above the defects of the three simulation models; b: the same functions as in Fig.a but in close up to region III, where a slight difference between the curves becomes visible

It is to note that in these simulations no heat loss due to radiation or convection was taken into consideration. Additional FEM models have been calculated with consideration of heat loss due to radiation. Then in region II the temperature plateau is not constant any more, but slightly decreasing. But this does not change the comparison results of the flat bottom hole and occluded defects.

For the evaluation of the flash thermography results different data processing algorithms are used. It has been already shown in several publications [3] that in this way much better signal-to-noise ratio can be achieved than with evaluating only one single thermographic image. Two methods are compared in this paper: PPT [4] and TSR [5].

In PPT method the numerical version of the Fourier transformation, FFT is used to transform the temperature time data into the frequency domain. Strictly taken, the FFT calculation only for periodical functions or for functions limited in time may be used. Hence this is not valid for the flash heating test, the measurement parameters, as sampling frequency or time duration should be selected carefully, as they strongly influence the obtained phase images [6]. Fig.5a compares the phase contrast between defect position and sound surface, calculated for the three above described simulation models. As the temperature vs. time functions differ only in the late time, i.e. in region III, therefore the phase functions show a difference only for the very low frequencies. Usually, in the PPT evaluations either the negative minimum, which is in absolute value the largest, or the positive maximum after the zero-crossing frequency are 
investigated [6]. In both these cases, no difference can be observed between a FBH defect and an inclusion filled with air.

In TSR testing, after a polynomial fitting [3], the $1^{\text {st }}$ or $2^{\text {nd }}$ derivative images are investigated. It is known [7], that the $2^{\text {nd }}$ derivative has the first maximum at $t=t_{w}$. As at this early time there is no difference between the temperature versus time functions, there is also no difference between the first maxima of their $2^{\text {nd }}$ derivatives, see Fig. $5 \mathrm{~b}$. A slight difference can be observed between the minima of the $2^{\text {nd }}$ derivatives, which correspond to the time $t_{c}$, i.e. the transition time between region II and region III.

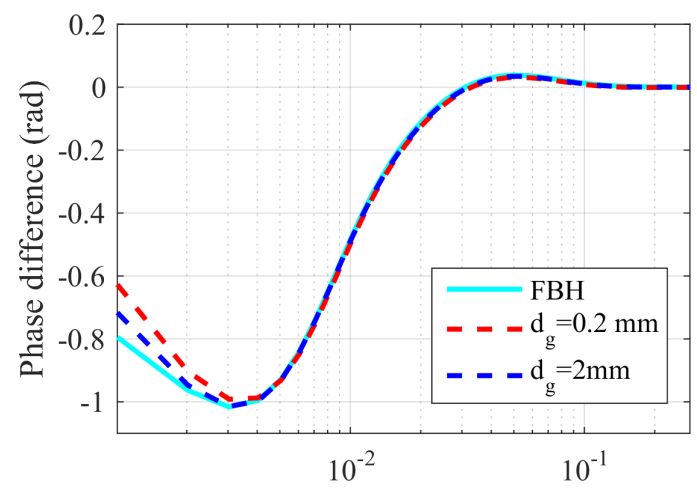

Frequency $(\mathrm{Hz})$ a)

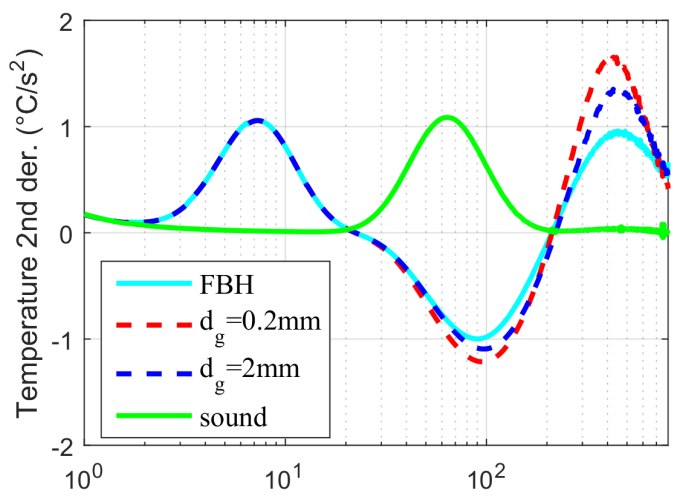

Time (s) b)

Figure 5: Phase difference between defect position and sound surface (a) and $2^{\text {nd }}$ derivative (b) for the three compared simulation models

If the lateral size of the defect is small, then the region II may almost diminish and no horizontal temperature plateau can be observed. In Fig.6a is such a model depicted: the diameter of the defect is $D=4 \mathrm{~mm}\left(d=2 \mathrm{~mm}, d_{g}=0.2 \mathrm{~mm}\right)$. In Fig.6b the temperature functions for the FBH defect and the models containing inclusions are compared and no relevant difference between the defects can be observed.

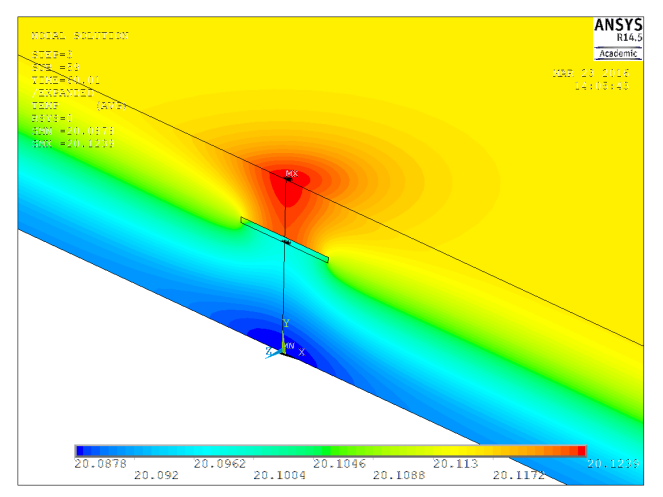

a)

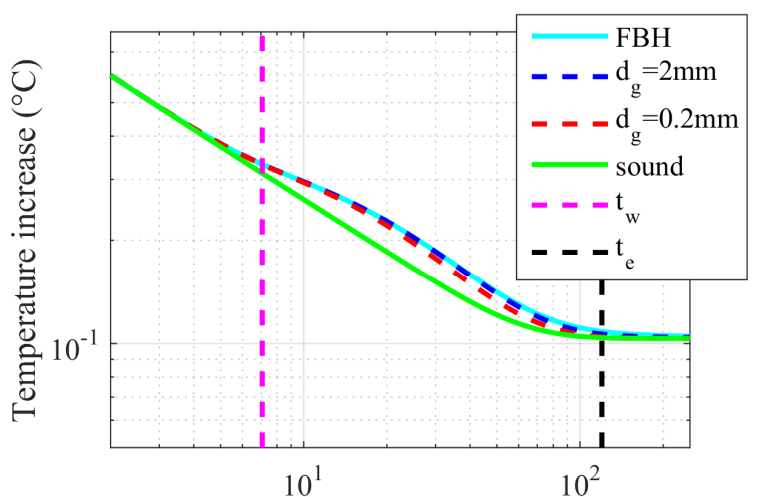

Time (s) b)

Figure 6a: Calculated temperature distribution for an FEM model: $D=4 \mathrm{~mm}, d=2 \mathrm{~mm}, d_{g}=0.2 \mathrm{~mm}, d_{\text {sound }}=6 \mathrm{~mm}$; $b$ : temperature versus time plots comparing FBH defect and models with air gaps with two different heights

Calculating the phase for these models with the small defect diameter, a slight difference can be observed, if the air gap is only $0.2 \mathrm{~mm}$ high, compared to the other two models, see Fig.7a. This difference occurs at low frequencies, because the temperature curves distinguish only in later time. In the TSR evaluation the $2^{\text {nd }}$ derivatives are mainly identical for all the three models, no relevant difference can be observed, see Fig. $7 \mathrm{~b}$.

These models have been simulated with material parameters for plastic, which means low heat conductivity and therefore long time duration until the temperature is equilibrated over the sample. But exactly the same behavior can be observed, if the heat conductivity is high, e.g. for steel, which material parameters are also included in Table 1. The thermal diffusivity is about two magnitudes higher than for plastic, therefore the time duration $t_{\text {end }}$ is set much shorter and the sampling frequency much higher (see Tab.1). In order to compare the results for two geometrically identical models with different materials, the temperature curves have been normalized. In Fig. 8 on the $x$-axis $\sqrt{t \kappa \pi}$ is plotted instead of $t$. Therefore, if $t=t_{w}$, then $x_{w}=\sqrt{t_{w} \kappa \pi}=d$, independently on the material parameters. Hence on the $y$-axis $T \lambda / Q \kappa$ is plotted instead of $T$, therefore if $T=T_{\text {end }}$, then the curves have a value of $1 / d$, independently on the material parameters. With this normalization the temperature curves can be well compared for different materials, and they are identical (see Fig. 8), as long as the effusivity ratios of the main material and the included one (in this case air) remain similar, which 
topic is investigated in Section 5. Therefore, the previous considerations regarding flat bottom holes and air gaps are not only for plastics but are also valid for other materials with isotropic material properties.
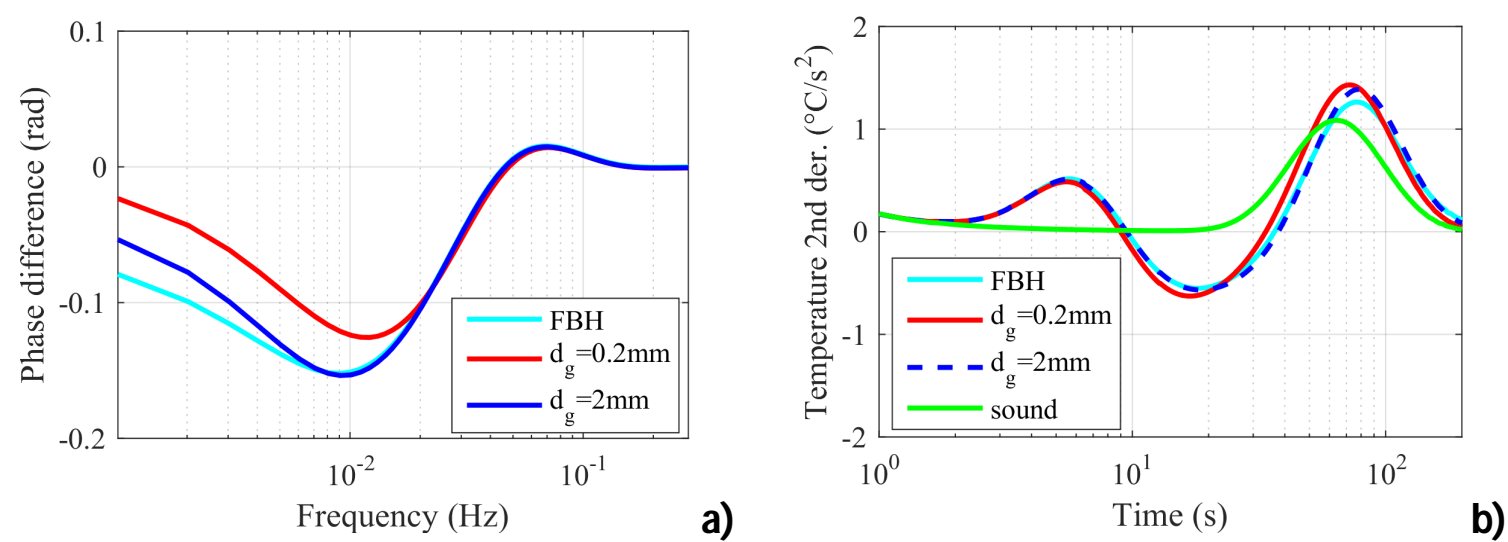

Figure 7: Phase (a) and $2^{\text {nd }}$ derivative (b) calculated for the three models with small defect size $(D=4 \mathrm{~mm})$

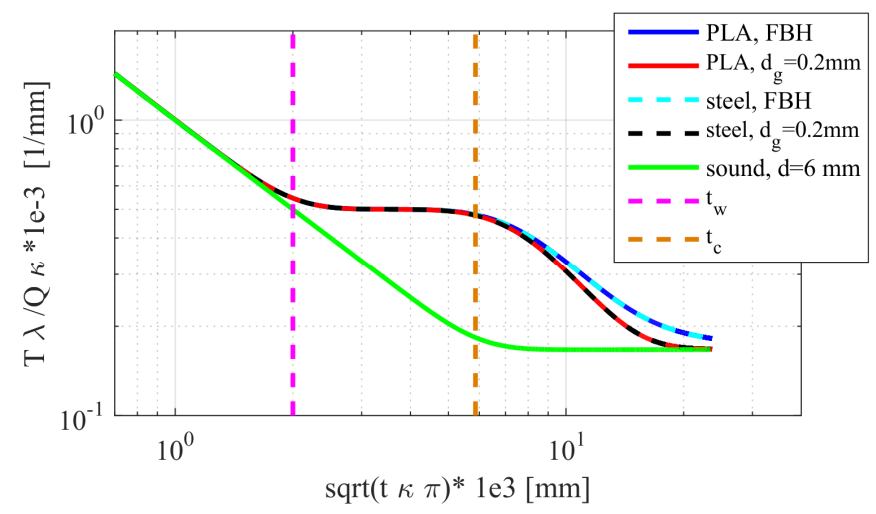

Figure 8: Normalized temperature curves showing identical plots for models from plastic and from steel.

\section{Measurement results}

3D printing of plastic parts presents an excellent possibility to produce samples with different geometries. Not only specimen with flat bottom hole but also a sample with an air inclusion can be printed. Fig. 9 shows these two samples with external sizes of $70 \times 50 \times 6 \mathrm{~mm}^{3}$ and the defect diameter is $20 \mathrm{~mm}$ in both of them. In the sample on the left side an air inclusion has been created, with the same diameter and in the same depth as the FBH defect at the right side. The specimens were 3D printed with Makerbot Replicator 2x printer from Polylactic acid plastic (PLA) with an infill density of $100 \%$. In Fused Deposition Modeling (FDM) 3D printing the model is built up in layers. Since every layer is on top of another one, this leads that the upper surface of a cavity has no support and the first few layers above the cavity can be slightly sagging, see Fig.10a. After cutting a sample through the cavity the created hole can be well investigated, and this sagging is mainly negligible, see Fig.10b.

Building up the specimen layer by layer with a thin extruded wire and an infill of $100 \%$, requires a 3D printer with good settings for the slicing software and for the printer itself. Even the slightest misadjustment results in material excess or small air holes in the sample. It must also be considered, that the PLA polymer used for printing the specimen is colored by pigments that influences the translucency of the polymer. After comparing PLA materials with different colors and with different compounds, we have used a black PLA, which is not transparent in the infrared spectrum of the camera $(1.5-5.2 \mu \mathrm{m})$; therefore no additional black painting was necessary for the flash measurements. 

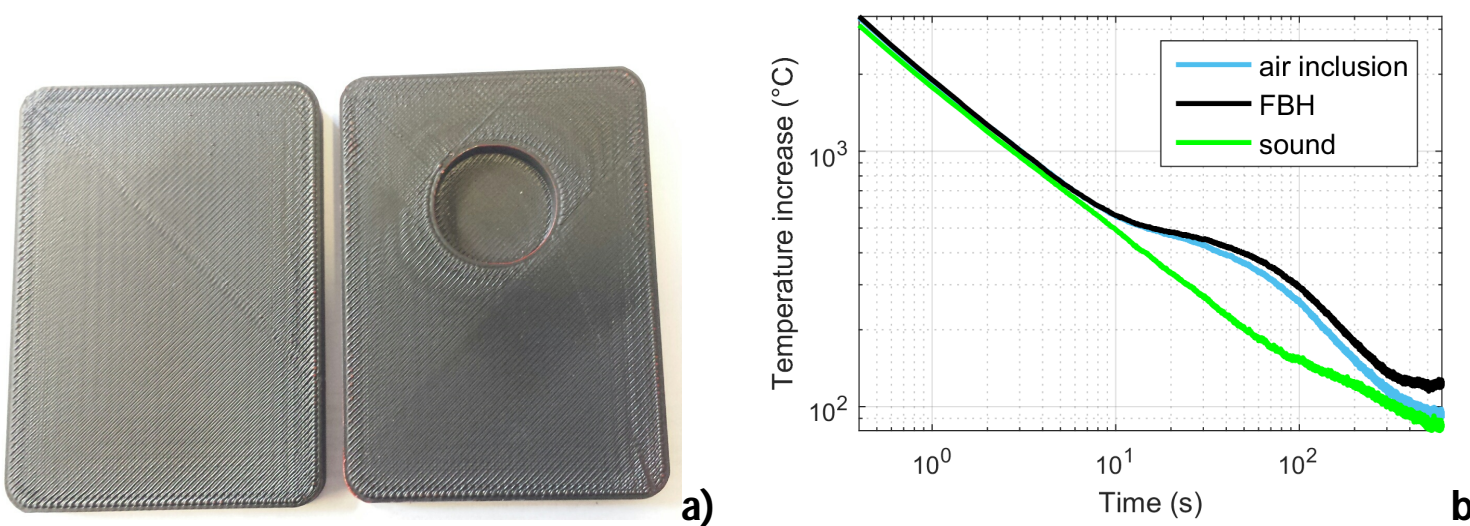

Figure 9: a) Two plastic samples produced with 3D printing, in the left one an air inclusion has been produced at the same position and in the same size as in the right one with the flat bottom hole; b) temperature versus time plots recorded for these two samples after a flash pulse.
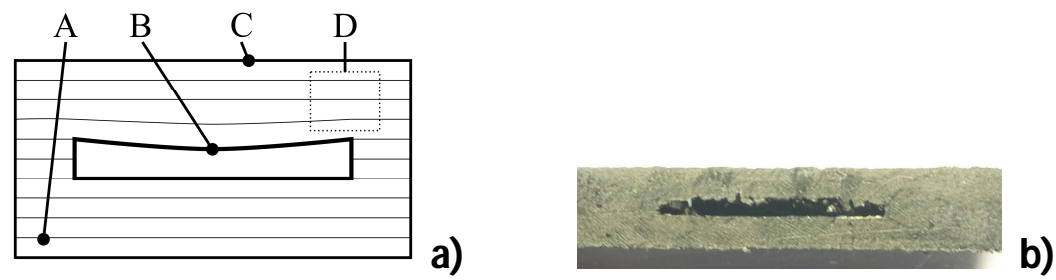

Figure 10: a) Sketch of 3D printed specimen with a cavity inside. Following problem zones are marked: A) Specimen built layer-by-layer that leads to an inhomogeneous structure; B) sagging of unsupported structure; C) roughness of the surface; $D$ ) transparency of polymer depending on plastic type and additional color-pigments; $b)$ Photo of a sample after cutting through the cavity, nominal parameters of the sample: entire thickness=5mm, cavity height= $1 \mathrm{~mm}$, material thickness above and below the defect $=2 \mathrm{~mm}$.

Fig.11a shows the phase images for these two samples at the frequency of $0.00335 \mathrm{~Hz}$. The phase difference has its minimum at this frequency, similarly to the FEM simulation results shown in Fig.5a. In both samples the defect is visible due to the lower phase value. In Fig. $11 \mathrm{~b}$ the phase contrasts between the midpoint above the defects and the sound surfaces are compared for these two samples. A slight difference between the curves can be observed, which can be rather explained by the not $100 \%$ reproducibility of the 3D printing and the measurement, than by the difference of the defects.

\section{$\mathrm{f}=0.00335 \mathrm{~Hz}$}
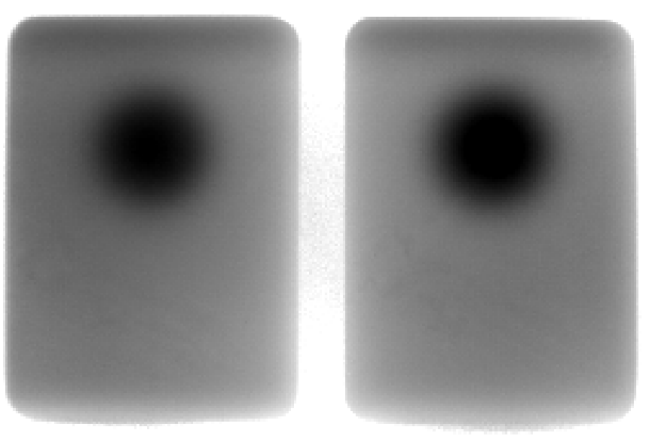

a)

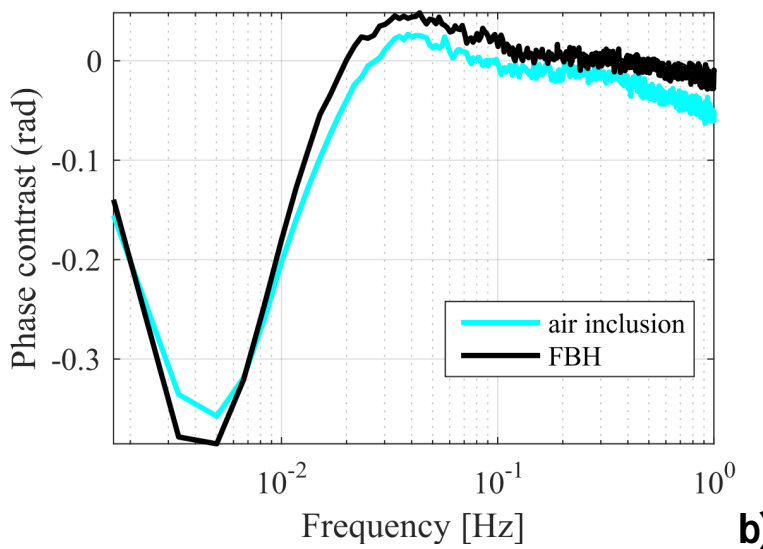

b)

Figure 11: a: Phase images of the two $3 D$ printed plastic samples at $f=0.00335 \mathrm{~Hz}$; left: air inclusion, right: $F B H$; $b$ : phase contrast versus frequency for these two samples

The same flash pulse measurement has been evaluated also with the TSR technique, and its results are presented in Fig.12. The $2^{\text {nd }}$ derivatives are mainly identical for both samples without any relevant differences. 

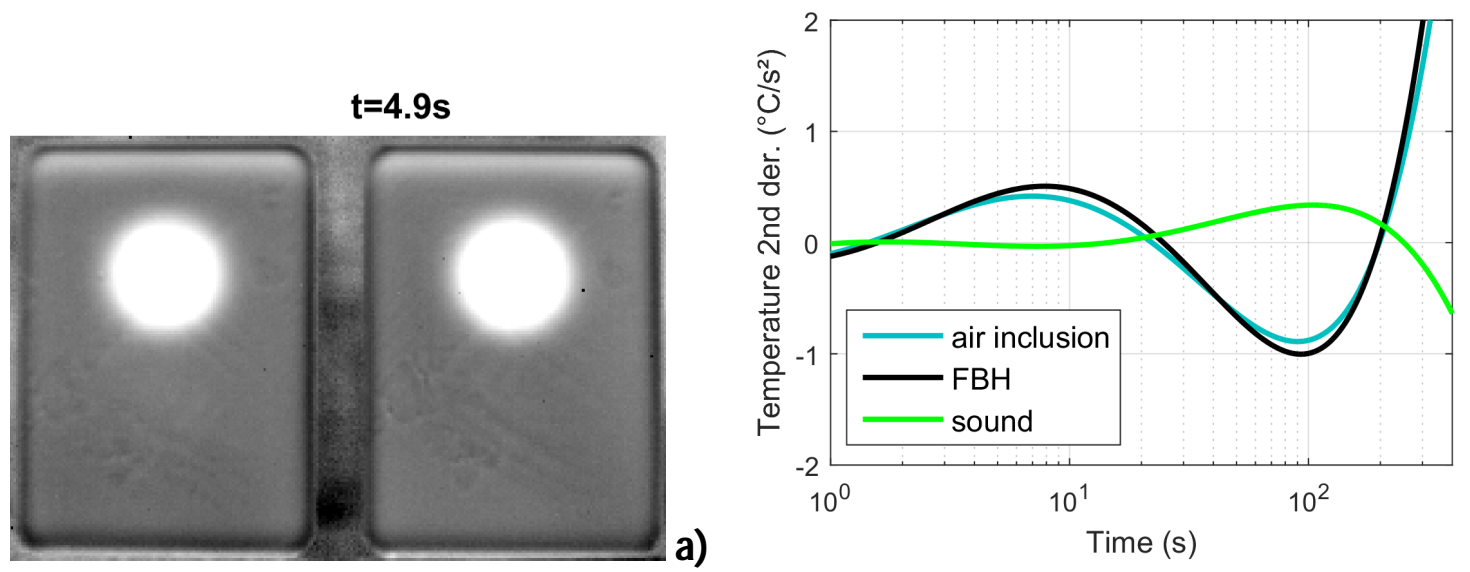

b)

Figure 12: a: $2^{\text {nd }}$ derivative image of the two $3 D$ printed plastic samples at $t=4.9 s$; left: air inclusion, right: $F B H$; $b: 2^{\text {nd }}$ derivative versus time for these two samples

\section{Simulation results for metal inclusion}

The situation is different, if the hole is not filled by air, but by another material, which changes the heat flow through the boundary separating both materials. A simulation model of a plastic sample has been setup, where the gap inside is filled with aluminum and an ideal heat conduction between the two materials has been assumed, see Fig.13a. The temperature versus time plots for the inclusions filled with air and with aluminum are compared in Fig.13b. In region II the heat is not trapped above the metal inclusion, but it is conducted due to its high heat conductivity into the region below, therefore the temperature is decreasing quicker above the defect than at the sound surface.

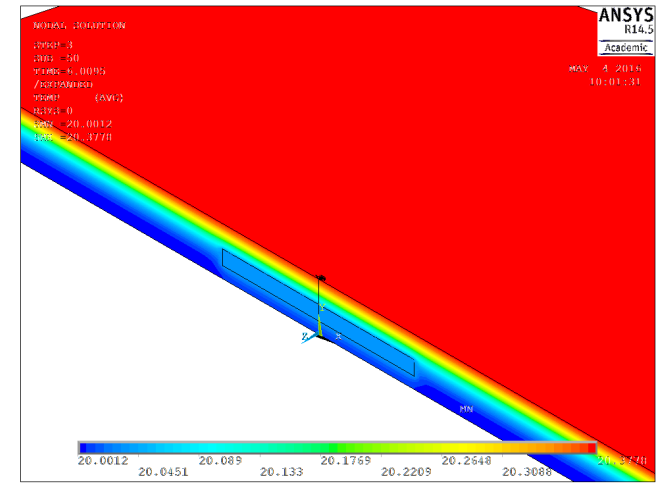

a)

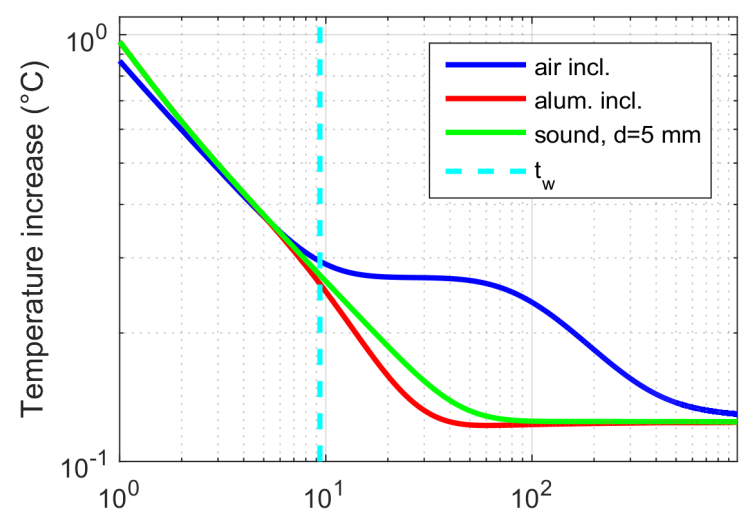

Time (s) b)

Figure 13: a: Simulated temperature distribution a couple of seconds after a short pulse heating for a model of a plastic sample with a hole including aluminum; $b$ : temperature versus time plots comparing models with inclusions filled with air and with aluminum ( $\left.D=20 \mathrm{~mm}, d=2.3 \mathrm{~mm}, d_{g}=1.5 \mathrm{~mm}, d_{\text {sound }}=5 \mathrm{~mm}\right)$

It has been shown $[8,9]$ that in the case of a harmonic excitation the difference between the phase of the excitation wave and the phase of a thermal wave at the surface of a finite body with thickness $d$ can be expressed as:

$$
\Phi_{\text {thwave }}=\arctan \left[\frac{-R_{b}\left(1+R_{g}\right) e^{-x} \sin (x)}{1-R_{g}\left(R_{b} e^{-x}\right)^{2}+R_{b}\left(1-R_{g}\right) \cos (x)}\right]
$$

where

$$
x=\frac{2 d}{\mu}=\frac{2 d}{\sqrt{\frac{2 \kappa}{\omega}}}=\sqrt{\frac{2 \omega}{\kappa}} d
$$


and $\mu$ is the thermal diffusion length of the wave with the angular frequency $\omega$. In order to calculate the phase contrast at the surface of a sample containing a defect, the phase difference between the part containing the defect in depth $d$ and the sound part with thickness $d_{s}$ has to be taken into count:

$$
\Delta \Phi_{\text {contrast }}=\Phi_{\text {th.wave }}\left(R_{g}, R_{b}, d\right)-\Phi_{\text {th.wave }}\left(R_{g}, R_{g}, d_{s}\right)
$$

The reflection of thermal waves on the boundary of two materials depends on their effusivity ratio [9]:

$$
\begin{aligned}
& b=\frac{\sqrt{\rho_{2} c_{2} \lambda_{2}}}{\sqrt{\rho_{1} c_{1} \lambda_{1}}} \\
& R_{b}=\frac{1-b}{1+b}
\end{aligned}
$$

With the material parameters summarized in Tab.1, it results in a reflection factor of $R_{b}=-0.93$ for plastic to aluminum. For plastic - air is a reflection factor of 1 assumed: $R_{g}=1$. Fig. 14 a compares the phase difference calculated for thermal waves by Eq. 3 and the phase calculated for the FEM simulation results using FFT. For the air inclusion the phase trends of the thermal wave and of the simulated PPT result look similar, the difference occurs only at low frequencies. This deviation has been investigated in another publication [6]. For aluminum inclusion $\left(R_{b}=-0.93\right)$ the difference between the phases calculated for thermal wave and for a defect using FFT is more significant and shows a relevant discrepancy between these two calculation techniques. This question will be investigated in a separate paper.
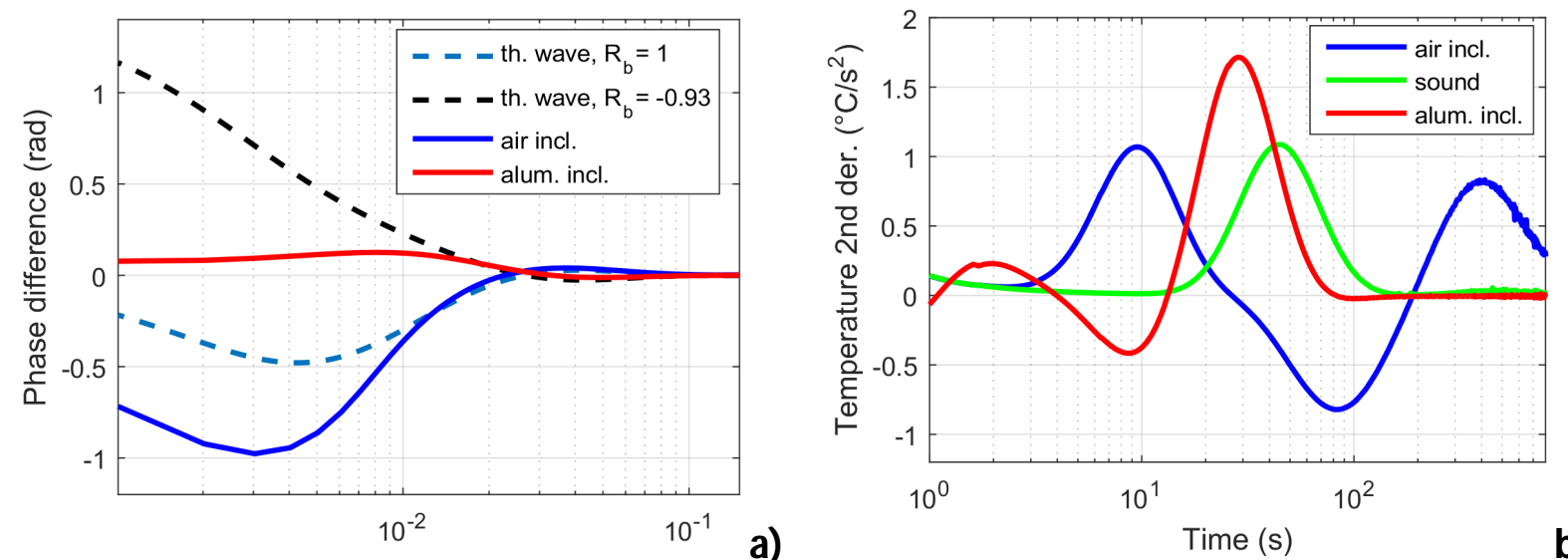

Figure 14: Temperature vs. time functions (a) phase contrast (b) for the three previously shown simulation models

Fig.14b compares the $2^{\text {nd }}$ derivatives for an air inclusion and for an inclusion filled with aluminum. The air inclusion shows a first maximum at $t=t_{w}$, as it has been already demonstrated in Fig. $5 \mathrm{~b}$. On the other hand, the aluminum inclusion has a negative $2^{\text {nd }}$ derivative and a minimum at $t=t_{w}$, as the temperature decreases quicker than at the sound surface, see also in Fig. 13b. These results are in very good agreement with the ones published by Shepard [7].

\section{Measurement results for metal inclusions}

We could manage to create a specimen with $3 \mathrm{D}$ printing, where the plastic sample contains as well an aluminum as an air inclusion. The printing process has been stopped and in one of the two prepared holes a $1.5 \mathrm{~mm}$ thick plate of aluminum has been placed, see Fig.15a. The metal plate was preheated before insertion into the specimen during the print process, to achieve good surface contact and a good heat transfer between plastic and metal, and to avoid an air gap between the metal insert and the plastic. After this step the 3D printing has been continued and as well the metal insert as the air gap has been covered with a further plastic layer.

Fig.16a shows phase image at $0.005 \mathrm{~Hz}$. In good agreement with the theory and with simulation results (Fig.14a), the surface above the hole filled with air has lower phase and above the aluminum plate has higher phase value than the sound surface.

The same measurement is evaluated also with TSR technique and the results are depicted in Fig.17. It shows the $2^{\text {nd }}$ derivative image at $t=5.3 \mathrm{~s}$. In good agreement with the theory and with simulation results (Fig.14b), the surface above the hole filled with air has a positive maximum at $t=t_{w}$ and above the aluminum plate it has a minimum. 

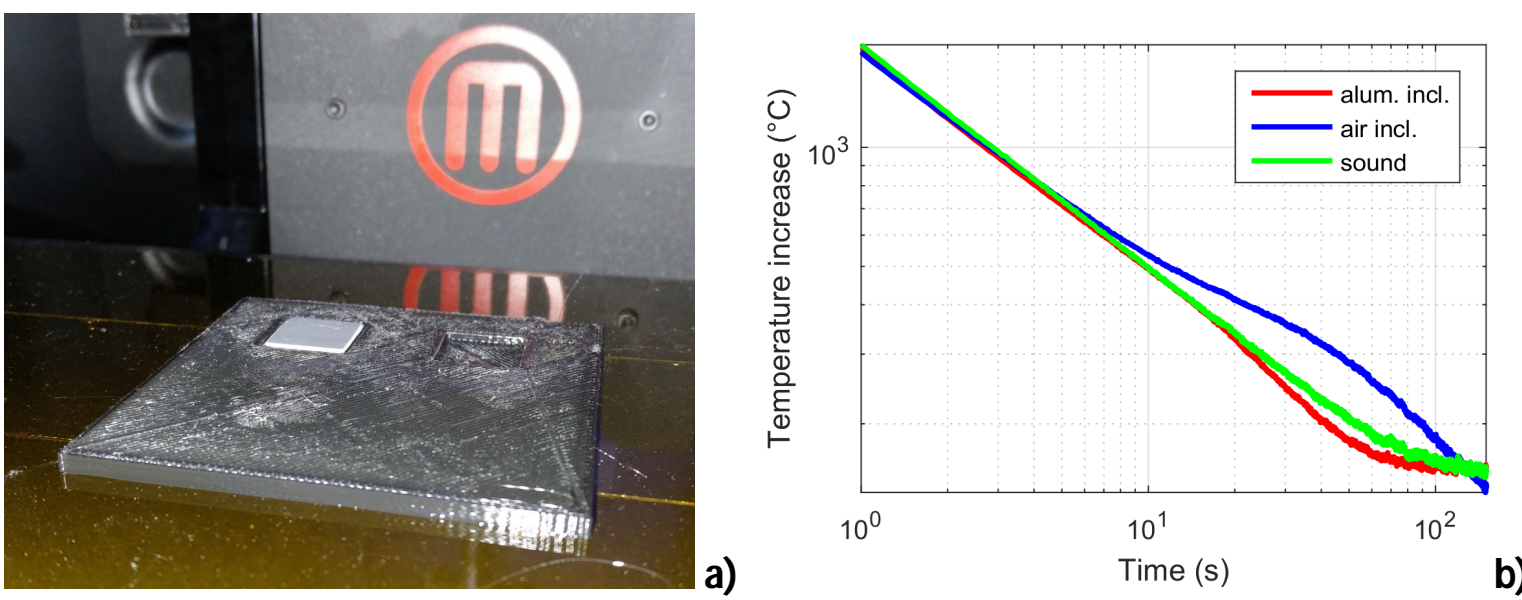

Figure 15: a: Plastic sample during 3D printing, in the inclusion of the left side an aluminium plate has been placed, the other inclusion with the same dimension contains air; $b$ : temperature versus time plots recorded after a short flash pulse for the completed sample, when the inclusions are covered with further plastic layers.

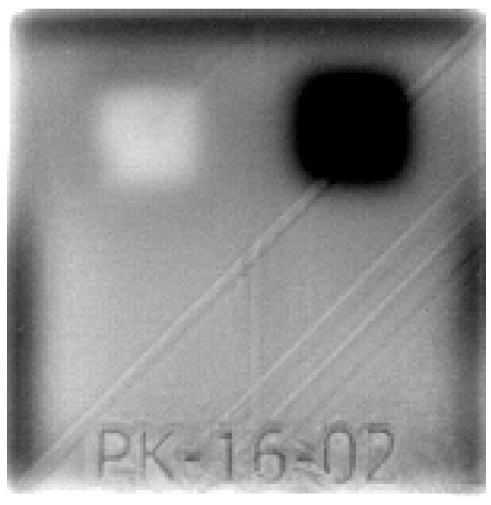

a)

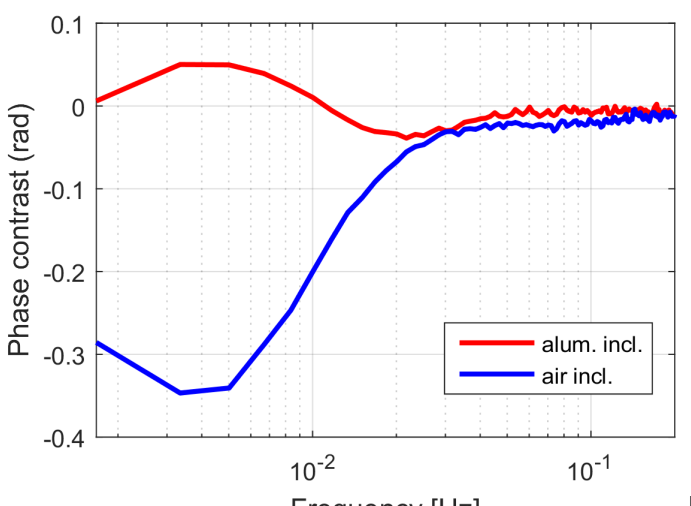

Frequency $[\mathrm{Hz}]$

b)

Figure 16: PPT results for the sample with two inclusions: the left one contains aluminium and the right one air; a: phase image at $f=0.005 \mathrm{~Hz}$; : phase contrast versus frequency for both inclusions.
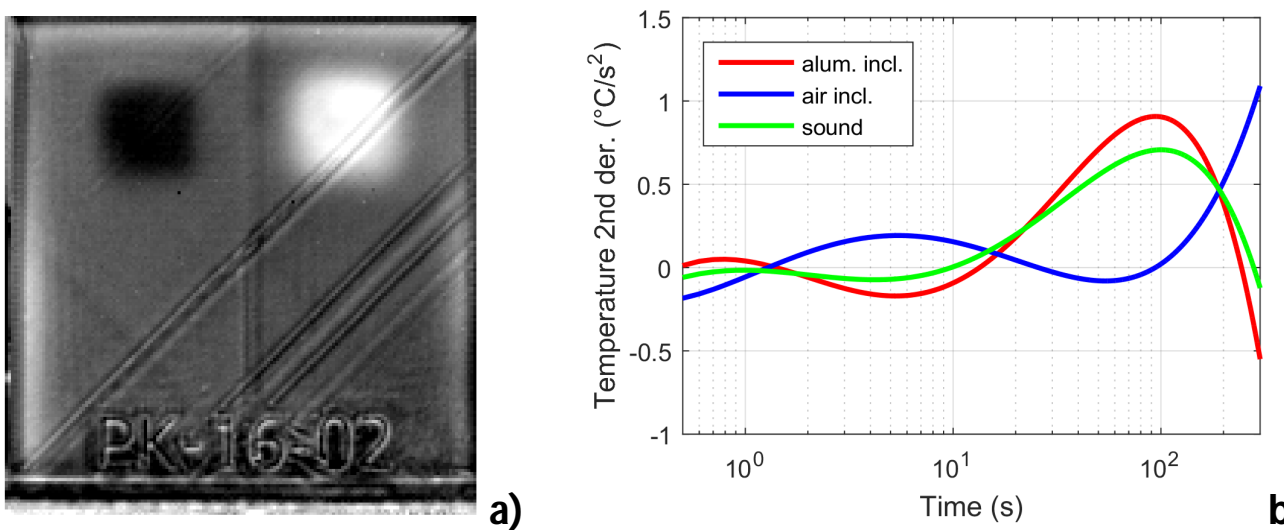

Figure 17: TSR results for the same measurement as also evaluated in Fig. 16. a: $2^{\text {nd }}$ derivative image at $t=5.2 s ; b: 2^{\text {nd }}$ derivative curves versus time for both inclusions.

\section{Summary}

It has been shown, that mainly the same thermographic results can be obtained with flat bottom hole defects and with inclusions filled with air. In many cases flash measurements are evaluated with PPT or with the TSR technique, and the usual evaluation images will not show any differences between these two types of defects. In PPT a difference 


\subsection{1/qirt.2016.097}

occurs only for very low frequencies, which are usually not calculated any more, as they require very long measurement duration. By TSR usually the $1^{\text {st }}$ maximum of the $2^{\text {nd }}$ derivative is investigated, which is identical for both types of defects. In order to distinguish them, the further maxima or minima of the $2^{\text {nd }}$ derivatives should be compared.

3D printing represents an excellent possibility to create samples with artificial defects. We have printed plastic samples with flat bottom holes and with air inclusions. The measurement results on these samples are in good agreement with the simulation results.

We have also printed a plastic sample including an aluminum plate and also an air inclusion. As the measurement results correspond very well with the theoretical ones, it proves that the 3D printing was in fact successful, and it can be excellently used to produce samples with artificial defects.

\section{REFERENCES}

[1] Carslaw, H.S., Jaeger J.C., [Conduction of Heat in Solids], Oxford University Press, Oxford, (1959).

[2] ANSYS Inc., http://www.ansys.com

[3] Rothbart N. et al, "A round robin test of flash thermography of CFRP and metal structures," Proc. $7^{\text {th }}$ Int. Symposium on NDT in Aerospace, Bremen, (2015)

[4] Maldague X., Marinetti S., "Pulse Phase Infrared Thermography”, J. Appl.Phys., Vol. 79, No. 5, pp. 2694-2698, 1996.

[5] Shepard, S.M., "Temporal noise reduction, compression and analysis of thermographic image data sequences," U.S. Patent 6516084, (2003)

[6] Oswald-Tranta B., "Comparison of time and frequency behavior in TSR and PPT evaluation", in Proc. SPIE, Thermosense XXXVIII, vol.9861 (2016).

[7] Shepard, S.M., Beemer, M.F., "Multilayer material characterization using thermographic signal reconstruction", AIP Conf. Proc. 1706, 100005 (2016); http:// dx.doi.org/10.1063/1.4940565

[8] Rosencwaig A., Gersho A., "Theory of the photoacoustic effect with solids," J. Appl. Phys. 47, 64 (1976).

[9] Bennett C.A, Patty R.R, "Thermal wave interferometry: a potential application of photoacoustic effect," Applied Optics, Vol.21, No1, (1982). 